\title{
SELECTION THEOREMS AND INVARIANCE OF BOREL POINTCLASSES
}

\author{
SREELA BHATTACHARYA AND S. M. SRIVASTAVA
}

\begin{abstract}
We generalize some known selection theorems and give simple proofs of results on the invariance of Borel pointclasses obtained by SaintRaymond, Jayne and Rogers, and Kunen and Miller.
\end{abstract}

1. Introduction. In [9], Saint-Raymond proved the following selection theorem.

THEOREM 1.1. Suppose $X$ and $Y$ are compact metrizable spaces and $Z$ is a second countable metrizable space. If $f: X \rightarrow Y$ is a continuous surjection and $p: X \rightarrow Z$ is a Borel measurable function of class $\alpha$, then there is a class 1 map $s: Y \rightarrow X$ such that $p \circ s$ is of class $\alpha$ and $f(s(y))=y$ for all $y$.

Jayne and Rogers $[3,4]$ have made a detailed study of this result and have extended it to the nonseparable case for continuous as well as for class 1 maps. Here we extend the result of Saint-Raymond in the more general set-up of Kuratowski and Ryll-Nardzewski [7] and Debs [1]. Our proof is simpler than those of SaintRaymond, Jayne and Rogers.

As an application Saint-Raymond, Jayne and Rogers gave results on the complexity of preimages of Borel sets. For the sake of completeness we indicate these applications in our paper.

2. Notation and preliminaries. Throughout $T$ will denote an arbitrary set, $\mathfrak{A}$ a family of subsets of $T$, and $X, Y, Z$ second countable metrizable spaces. A second countable, completely metrizable space is called a Polish space. Given $\mathfrak{A}, \mathfrak{A}_{\sigma}\left(\mathfrak{A}_{\delta}\right)$ will denote the family of unions (intersections) of a sequence of sets in $\mathfrak{A}$.

A multifunction $F: T \rightarrow X$ is a map defined on $T$ whose values are nonempty subsets of $X$. For $E \subseteq X$,

$$
F^{-1}(E)=\{t \in T: F(t) \cap E \neq \varnothing\} .
$$

We say that $F$ is $\mathfrak{A}$-measurable (strongly $\mathfrak{A}$-measurable) if $F^{-1}(E) \in \mathfrak{A}$ for every open (closed) set $E$ in $X$. In particular, a point map $f: T \rightarrow X$ is $\mathfrak{A}$-measurable if $f^{-1}(E) \in \mathfrak{A}$ for every open subset $E$ of $X$. A function $s: T \rightarrow X$ is called a selector for $F$ if $s(t) \in F(t)$ for every $t \in T$. The set

$$
\{(t, x) \in T \times X: x \in F(t)\}
$$

is called the graph of $F$ and is denoted by $\operatorname{Graph}(F)$. If $T$ is a topological space then $F: T \rightarrow X$ is called lower semicontinuous (upper semicontinuous) if $F^{-1}(A)$ is open (closed) for every open (closed) set $A$ in $X$.

Received by the editors June 21, 1985.

1980 Mathematics Subject Classification. Primary 03E15, 54C60, 54C65.

Key words and phrases. Set-valued maps, measurable selections, Borel sets. 
For notation and terminology in descriptive set theory we follow Kuratowski [6]. The set of natural numbers will be denoted by $\omega$. Further, $\omega^{<\omega}=\bigcup_{k \in \omega} \omega^{k}$. We shall use $e$ to denote the empty sequence. A map $f: X \rightarrow Y$ is called an open (closed) map if $f(X)=Y$ and the image of every open (closed) set in $X$ is open (closed) in $Y$. For $A \subseteq X, \operatorname{cl}(A)$ will denote the closure of $A$ in $X$.

3. Main results. From now on, $\mathcal{L}$ is a field of subsets of $T, X$ and $Y$ are Polish spaces and $Z$ is a second countable metrizable space.

THEOREM 3.1. If $F: T \rightarrow X$ is a closed valued, strongly $\mathcal{L}_{\sigma}$-measurable multifunction and $g: X \rightarrow Z$ is a class 1 map, then there is an $\mathcal{L}_{\sigma}$-measurable selector $s: T \rightarrow X$ for $F$ such that $g \circ s$ is also $\mathcal{L}_{\sigma}$-measurable.

ProOF. Fix a complete metric $d$ on $X$ compatible with its topology such that $d$-diameter $(X)<1$.

Step 1. There are systems of subsets $\left\{T(s): s \in \omega^{<\omega}\right\}$ and $\left\{H(s): s \in \omega^{<\omega}\right\}$ of $T$ and $X$ respectively such that, for every $s \in \omega^{k}$ and $n, m \in \omega(n \neq m)$,

(i) $T(s) \in \mathcal{L}_{\sigma}, T(e)=T$,

(ii) $T(s n) \cap T(s m)=\varnothing$,

(iii) $T(s)=\bigcup_{j \in \omega} T(s j)$,

(iv) $H(s)$ is closed and of $d$-diameter $<2^{-k}$,

(v) $d^{\prime}$-diameter $(g(H(s)))<1 / 2^{k}$ (where $d^{\prime}$ is a fixed metric on $Z$ compatible with its topology),

(vii) for every $t \in T(s), F(t) \cap H(s) \neq \varnothing$.

To see that such systems exist we shall proceed inductively. Define $T(e)=T$ and $H(e)=X$. Suppose for every $s \in \bigcup_{i=0}^{k} \omega^{i}, T(s)$ and $H(s)$ have been defined satisfying (i)-(vii). Fix an $s \in \omega^{k}$. Fix a base $W_{0}, W_{1}, \ldots$ for $Z$ such that $d^{\prime}$ $\operatorname{diameter}\left(W_{i}\right)<2^{-(k+1)}$ for all $i$. Also, fix a base $V_{0}, V_{1}, \ldots$ for $X$ such that, for each $i, d$-diameter $\left(V_{i}\right)<2^{-(k+1)}$.

Let $g^{-1}\left(W_{i}\right)=\bigcup_{j \in \omega} E_{i j}$, where $E_{i j}$ are closed in $X$. Enumerate $\left\{E_{i j}: i \in \omega, j \in\right.$ $\omega\}$ in a single sequence $F_{0}, F_{1}, \ldots$ For $m, n \in \omega$, let

$$
T^{\prime}(s)(n, m)=\left\{t \in T(s): F(t) \cap H(s) \cap \operatorname{cl}\left(V_{m}\right) \cap F_{n} \neq \varnothing\right\} .
$$

Since $F$ is strongly $\mathcal{L}_{\sigma}$-measurable and $T(s) \in \mathcal{L}_{\sigma}, T^{\prime}(s)(n, m) \in \mathcal{L}_{\sigma}, \forall m, n \in \omega$. Enumerate $\left\{T^{\prime}(s)(n, m): m, n \in \omega\right\}$ in a single sequence $\left\{T^{\prime \prime}(s)(n): n \in \omega\right\}$. By [7] we get pairwise disjoint sets $T(s n), n \in \omega$, in $\mathcal{L}_{\sigma}$ such that

$$
\bigcup_{n \in \omega} T(s n)=\bigcup_{n \in \omega} T^{\prime \prime}(s)(n)
$$

and

$$
T(s n) \subseteq T^{\prime \prime}(s)(n) \text { for all } n
$$

Put

$$
H(s n)=H(s) \cap \operatorname{cl}\left(V_{j}\right) \cap F_{i}
$$

whenever $T(s n) \subseteq T^{\prime}(s)(i, j)$. This completes the definition of two systems of sets with required properties.

Step 2. We now define the selector $s$ for $F$. Fix $t \in T$. There is a unique $\alpha \in \omega^{\omega}$ such that $t \in T(\alpha \mid k)$ for all $k$. By (iv) and (v), $\bigcap_{k \in \omega} H(\alpha \mid k)$ is a singleton. We define $s(t)$ to be the unique point of $\bigcap_{k \in \omega} H(\alpha \mid k)$. 
To prove that $s$ is $\mathcal{L}_{\sigma}$-measurable, fix a closed set $E \subseteq X$. Let

$$
E_{k}=\left\{x \in X:(\exists y \in E)\left(d(y, x)<1 / 2^{k}\right)\right\}, \quad k \in \omega .
$$

Also, note that for each $k$, the multifunction $F_{k}: T \rightarrow X$ defined by

$$
F_{k}(t)=F(t) \cap H\left(n_{0}, \ldots, n_{k-1}\right) \quad \text { if } t \in T\left(n_{0}, \ldots, n_{k-1}\right)
$$

is strongly $\mathcal{L}_{\sigma}$-measurable. It is easy to check that

$$
s^{-1}(E)=\bigcap_{k \in \omega}\left\{t \in T: F_{k}(t) \subseteq E_{k}\right\}
$$

Hence, $s^{-1}(E) \in \mathcal{L}_{\delta}$. Thus, $s$ is $\mathcal{L}_{\sigma}$-measurable.

Now, it only remains to show that $g \circ s$ is $\mathcal{L}_{\sigma}$-measurable. To see this choose a point $x\left(n_{0}, \ldots, n_{k-1}\right) \in H\left(n_{0}, \ldots, n_{k-1}\right)$ for each $\left(n_{0}, \ldots, n_{k-1}\right)$. Define $f_{k}: T \rightarrow$ $X$ by

$$
f_{k}(t)=x\left(n_{0}, n_{1}, \ldots, n_{k-1}\right) \text { if } t \in T\left(n_{0}, \ldots, n_{k-1}\right) .
$$

It is easy to see that $g \circ f_{k}$ is $\mathcal{L}_{\sigma}$-measurable. Further, by (v), $\left\{g \circ f_{k}\right\}_{k \in}$. converges uniformly to $g \circ s$. Hence, $g \circ s$ is $\mathcal{L}_{\sigma}$-measurable [7]. The proof is complete.

We now extend this theorem for $G_{\delta}$-valued multifunctions.

THEOREM 3.2. Let $T, X, Z, \mathcal{L}$ and $g$ be as in the previous theorem. Suppose $F: T \rightarrow X$ is a strongly $\mathcal{L}_{\sigma}$-measurable multifunction such that

$$
\operatorname{Graph}(F) \in(\mathcal{L} \times \mathcal{U})_{\sigma \delta}
$$

where

$$
\mathcal{L} \times \mathcal{U}=\{E \times U \subseteq T \times X: E \in \mathcal{L} \text { and } U \subseteq X \text { is open }\} .
$$

Then there is an $\mathcal{L}_{\sigma}$-measurable selector $s: T \rightarrow X$ for $F$ such that $g \circ s$ is also $\mathcal{L}_{\sigma}$-measurable.

Proof. Set $\operatorname{Graph}(F)=\bigcap_{k=1}^{\infty} G_{k}$, where $G_{k}=\bigcup_{n \in \omega}\left(E_{n k} \times U_{n k}\right), E_{n k} \in \mathcal{L}$ and $U_{n k}$ open in $X$, for all $n$ and $k$.

A slight modification of the argument in Step 1 of the previous theorem gives us a system of sets $\left\{T(s): s \in \omega^{<\omega}\right\}$ and $\left\{H(s): s \in \omega^{<\omega}\right\}$ in $T$ and $X$ respectively which satisfy conditions (i) - (vii) and

(viii) for every $k \in \omega, \bigcup_{s \in \omega^{k}}\{T(s) \times H(s)\} \subseteq G_{k}$.

Now we follow the arguments of Theorem 3.1 and obtain a selector $s$ with required properties.

REMARK. The set up of Theorem 3.2 is very similar to that of the main theorem in $[\mathbf{1}]$.

4. Selection theorems for lower semicontinuous and upper semicontinuous multifunctions. As a corollary to Theorem 3.1, we now give several generalizations to the selection theorem of Saint-Raymond. See also $[\mathbf{3}, \mathbf{4}]$.

THEOREM 4.1. Let $X$ be a Polish space and let $T, Z$ be second countable completely metrizable spaces. Suppose $g: X \rightarrow Z$ is a Borel measurable function of class $\alpha\left(1 \leq \alpha<\omega_{1}\right)$ and $F: T \rightarrow X$ is an upper semicontinuous (u.s.c.) closed valued 
multifunction. Then there is a class 1 selector $s: T \rightarrow X$ for $F$ such that $g \circ s$ is of class $\alpha$.

PROOF. Since every u.s.c. closed valued multifunction admits a class 1 selector, the result is true for $\alpha>\omega_{0}$. To prove the result for finite $\alpha$, we proceed by induction.

Suppose $\alpha=1$. Take $\mathcal{L}$ to be the family of subsets of $T$ which are simultaneously $F_{\sigma}$ and $G_{\delta}$ and apply Theorem 3.1 .

Assume the result is true for $\alpha=m$. If $g$ is of class $(m+1)$ then there is a sequence of class $m$ function $g_{n}: X \rightarrow Z$ which converges pointwise to $g$. Define $h: X \rightarrow Z^{\omega}$ by

$$
h(x)=\left(g_{0}(x), g_{1}(x), \ldots\right), \quad x \in X .
$$

By induction hypothesis, we get a class 1 selector $s: T \rightarrow X$ for $F$ such that $h \circ s$ is of class $m$. Then $g \circ s=\lim _{n} g_{n} \circ s$, and hence is of class $(m+1)$.

REMARK. The induction argument above is due to Saint-Raymond.

THEOREM 4.2. The previous theorem is also true when $F$ is lower semicontinuous (l.s.c.).

PROOF. We need to prove the result for $\alpha=1$ only. By a result of Michael [8] there is a compact valued, u.s.c. multifunction $H: T \rightarrow X$ such that $H(t) \subseteq F(t)$ for all $t$. We use Theorem 4.1 for $H$ and $g$.

THEOREM 4.3. Let $X$ and $Y$ be Polish spaces and let $Z$ be a second countable metrizable space. If $f: X \rightarrow Y$ is a class 1 , closed map and $g: X \rightarrow Z$ of class $\alpha$, then there is a class 1 map $s: Y \rightarrow X$ such that

(i) $f(s(y))=y$ for all $y \in Y$,

(ii) $g \circ s$ is of class $\alpha$.

PROOF. As in the case of Theorem 4.1, we need to prove the result for $\alpha=1$ only.

Case $f$ is continuous. Consider the multifunction $F(y)=f^{-1}(y), y \in Y$. Apply Theorem 4.1 for $T=Y, F$ and $g$.

Case $f$ is of class 1 . Let $\tilde{X}$ be $\operatorname{graph}(f), \tilde{f}$ the projection $\pi_{Y}: \tilde{X} \rightarrow Y$ and $\tilde{g}=g \circ \pi_{X}$. By a result of Jayne and Rogers [4, Lemma 5], the map $f$ is closed. We now use the previous case, get a class 1 map $\tilde{s}: Y \rightarrow \tilde{X}$ such that $\pi_{Y}(\tilde{s}(y))=y$ for all $y$ and $\tilde{g} \circ \tilde{s}$ is of class 1 . Put $s=\pi_{X} \circ \tilde{s}$.

THEOREM 4.4. The previous theorm is true for an open class 1 map $f$ also.

ProOF. As in the previous theorem, we can assume $\alpha=1$ and $f$ continuous. By the theorem of Michael, there is an $X^{\prime} \subseteq X$ such that $f\left(X^{\prime}\right)=Y$ and the restriction of $f$ to $X^{\prime}$ is perfect (or proper). It follows that $X^{\prime}$ is Polish [2]. Now use the previous theorem for $f: X^{\prime} \rightarrow Y$ and $g: X^{\prime} \rightarrow Z$.

The idea of using projection to deduce the results for class 1 maps from that in continuous maps is due to Jayne and Rogers. However, we can use Theorem 3.2 to get the results for class 1 closed maps and extend the result of Michael for class 1 open maps.

5. Invariance of Borel pointclasses. As an application of our results, we deduce the invariance results for Borel pointclasses (obtained by Saint-Raymond, Jayne and Rogers, and Kunen and Miller [5]). 
THEOREM 5.1. Let $X, Y$ be Polish spaces and let $f: X \rightarrow Y$ be a class 1 surjection which is either open or closed. If $E \subseteq Y$ is such that $f^{-1}(E)$ is a Borel set of multiplicative class $\alpha$ or of additive class $\alpha$ then $E$ is of the same class as that of $f^{-1}(E)$.

PROOF. The proof is sufficient to prove the result for multiplicative class $\alpha$. So assume $E \subseteq Y$ and $f^{-1}(E)$ is of multiplicative class $\alpha$. Get a class $\alpha$ map $g: X \rightarrow[0,1]$ such that $g^{-1}(0)=f^{-1}(E)$. By our results, there is a class 1 map $s: Y \rightarrow X$ such that $g \circ s$ is of class $\alpha$ and $f(s(y))=y$ for all $y$. Hence, $E=(g \circ s)^{-1}(0)$ is of multiplicative class $\alpha$.

An examination of the proof shows that the above theorem is also true when $f$ is a closed map and $f^{-1}(y)$ is closed in $X$ for each $y \in Y$.

\section{REFERENCES}

1. G. Debs, Selection d'une multi-application a valeurs $\mathcal{G}_{\delta}$, Acad Roy. Belg. Bull. Cl. Sci 65 (1979), 211-216.

2. R. Engelking, General topology, PWN, Warsaw, 1977.

3. J. E. Jayne and C. A. Rogers, Invariance of Borel classes in metric spaces, Math. Ann. 263 (1983), 323-341.

4. - The invariance of absolute Borel classes, Convex analysis and applications, Research Notes in Math., No. 57, Pitman, London, 1982, pp. 118-151.

5. K. Kunen and A. N. Miller, Borel and projective sets from the point of view of compact sets, Math. Proc. Cambridge Philos. Soc. 94 (1983), 399-409.

6. K. Kuratowski, Topology, Vol. I, Academic Press, New York, 1966.

7. K. Kuratowski and C. Ryll-Nardzewski, $A$ general theorem on selectors, Bull. Acad. Polon. Sci. Sér. Sci. Math. 13 (1961), 397-403.

8. E. Michael, $A$ theorem on semi-continuous set-valued functions, Duke Math. J. 26 (1959), $647-651$.

9. J. Saint-Raymond, Fonctions Boreliennes sur un quotient, Bull. Soc. Math. France (2) 100 (1976), 141-147.

Statistics and Mathematics Division, Indian Statistical Institute, 203 B.T. ROAD, CALCUTTA 700 035, INDIA 